\title{
A simple, sturdy and cheap sensor for distance and force in spatially con- strained environments
}

\author{
D. Plohmann*, G. Ackermann ${ }^{*}$, S. Eibl ${ }^{*}$ (s.eibl@sensorik-bayern.de), C. Meurer ${ }^{*}$, H.-J. Möstl', \\ M. Huber", H. Steigerwald ${ }^{* \#}$ \\ ") Sensorik-Bayern GmbH, Regensburg; ") Strategische Partnerschaft für Sensorik e.V., Regensburg
}

Coil springs show a well-defined relation between externally applied force and spring length, the spring rate. Thus, if one of these parameters is known, the other parameter can be calculated easily. Measurement of either of these two parameters using intrinsic parameters, like DC and AC electric resistance, is not a new idea and bears the potential to turn the coil spring into a promising sensor. On top of it being simple and sturdy, a sensor made of a small coil spring could be used in applications with strong requirements to the spatial extension of the sensor. This could make the coil spring a solution for cases, where existing sensors need to cede. While the idea of making a coil spring sensor is old and the potential applications are promising, it has, due to the technical difficulties involved, never been made into a working prototype. We have completed this last step and want to present our results hereon.

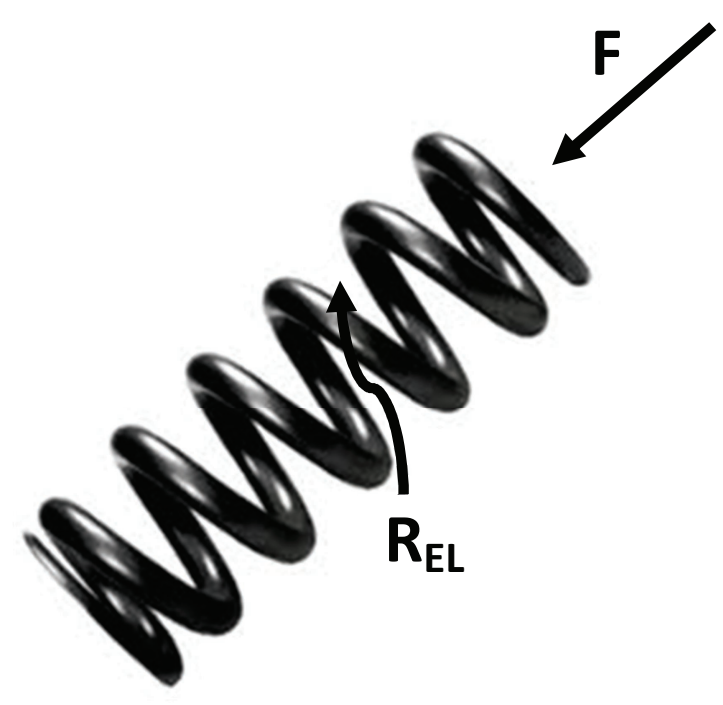

Figure 1: The AC/DC resistance of a coil spring is dependent on the spring load. We have exploited this fact to create a prototype of a new sensor concept for force and distance. 
The complex part of the resistance, the inductance, is to a certain extent dependent on the physical form of the coil - deformations of the coil are reflected in a change of inductance. Additionally, there is the proximity effect, which describes the change of the profile of the electrical flux in a conductor carrying alternating current in the proximity of other conductors. In the case at hand the neighbouring loops of the coil provide this influence, as the loops are pushed closer on compression of the spring. This effect is relevant to the complex as well as the real part of the resistance. The skin effect is of similar nature and describes the phenomenon of change in charge distribution from isotropic to superficial for high frequency alternating currents. These effects influence the electromagnetic behaviour of the coil spring in a complicated, but reproducible way and provide a basis for the determination of its length and therefore also the force it is subjected to.

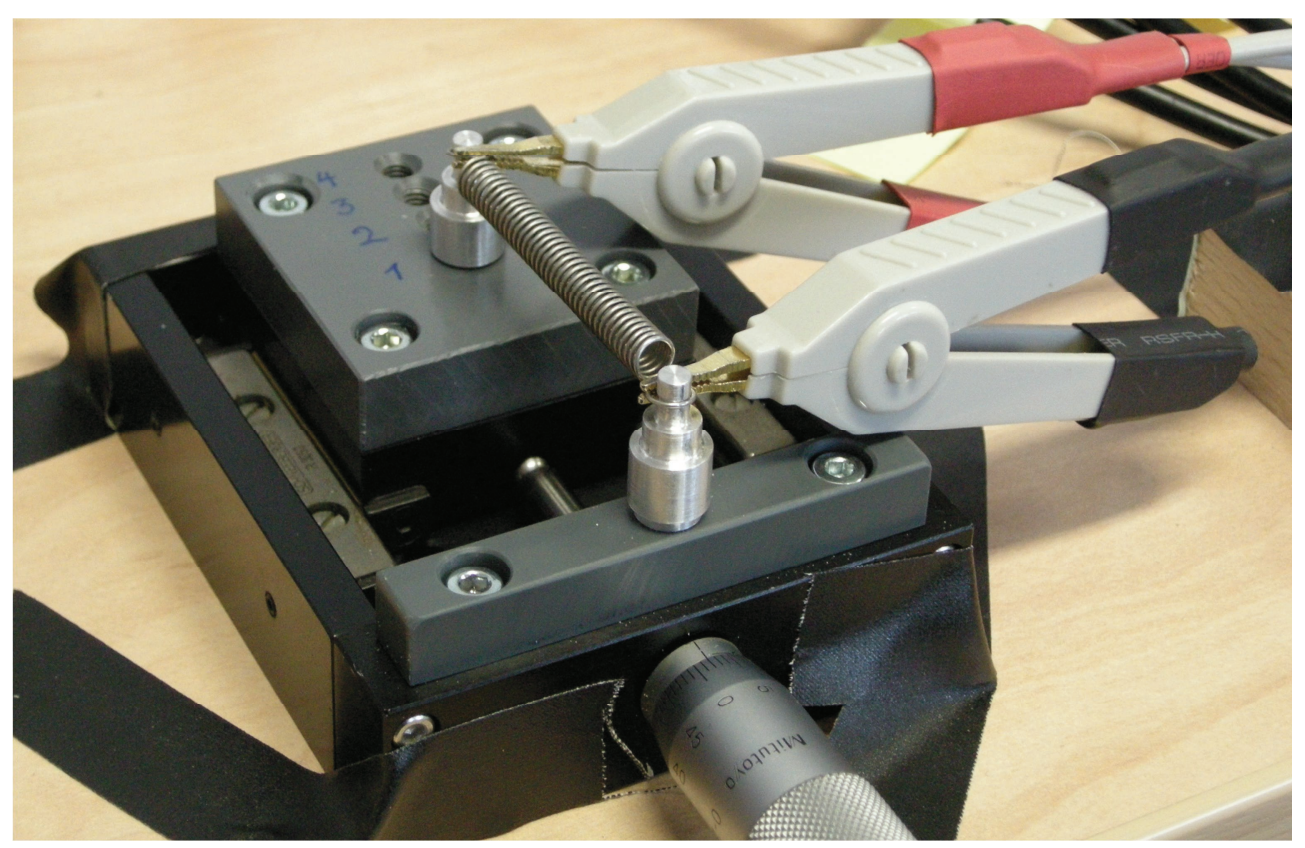

Figure 2: Experimental setup for determination of electromagnetic properties of the coil spring

Figure 2 shows a measurement for determination of the properties of a given coil spring. To probe the resistance of such a spring we use high frequency alternating current, in a way to best take advantage of the abovementioned effects and at the same time to minimize sensibility to uptake of external electromagnetic radiation. With our partners we have developed a sensor prototype using the coil spring as an AC/DC resistance in an RLC circuit. Here we exploit the variation of resonance frequency in the circuit with change in $A C / D C$ resistance of the spring. This solution does not require expensive measuring equipment and could make the coil spring part of an affordable device to build a cheap sensor. The main challenges we needed to overcome during implementation of the technique lie in the comparatively low electrical resistance of the spring, handling of high frequency alternating electrical currents and the mechanical properties of coil springs.

Our solution provides a prototype of a simple, sturdy and cheap sensor for distance and force with a high potential for opening up a new field of sensor solutions in spatially limited environments.

This project is supported by the "Zentrales Innovationsprogramm Mittelstand" (ZIM), support id: KF2374201WO9 